\title{
SASAKIAN ANTI-HOLOMORPHIC SUBMANIFOLDS OF A KAEHLER MANIFOLD
}

\author{
TEE-HOW LOO
}

\begin{abstract}
Let $M$ be a connected Sasakian anti-holomorphic submanifold of a Kaehler mnifold with flat norml connection and with $\operatorname{dim} D \geq 4$, where $D$ is the holomorphic distribution on $M$. We show that $M$ is locally Riemannian product $M^{\prime} \times M^{\prime \prime}$ where $M^{\prime}$ is homothetic to a Sasakian manifold and $M^{\prime \prime}$ is a locally Euclidean space.
\end{abstract}

\section{Introduction}

The concept of Saskian anti-holomorphic submanifolds of a Kaehler manifold was introduced by Bejancu [2] in analogy with the theory of Sasakian structure. Recently, Sun$\mathrm{Li}[6]$ adapted the notion of Sasakian anti-holomorphic submanifolds to CR-submanifolds and extended this study to Sasakian CR-submanifolds. They also proved that if $M$ is a Sasakian anti-holomorphic submanifold of a Kaehler manifold $N$ with flat normal connection and if $\operatorname{dim} D \geq 4$, where $D$ is the holomorphic distribution on $M$, then the $D$-mean curvature vector $H_{D}$ is parallel. Using this fact, we show that under certain conditions, a Sasakian anti-holomophic submanifold is locally a Riemannian product $M^{\prime} \times M^{\prime \prime}$, where $M^{\prime}$ is homotheic to a Sasakian manifold and $M^{\prime \prime}$ is a locally Euclidean space (cf. Theorem 3.9).

This work was done under the supervision of Dr. S. H. Kon at the University of Malaya and formed part of the author's thesis submitted for the M. Sc. degree.

\section{Preliminaries}

Let $N$ be an $n$-dimensional Riemannian manifold and let $M$ be an $m$-dimensional manifold isometrically immersed in $N$. Denote by $\langle$,$\rangle both the Riemannian metric of N$ and $M, \tilde{\nabla}$ the Levi-Civita connection on $N$ and $\nabla$ the connection induced on $M$. Then the Gauss and Weingarten formulas are given respectively by

$$
\begin{aligned}
\tilde{\nabla}_{X} Y & =\nabla_{X} Y+h(X, Y) \\
\tilde{\nabla}_{X} Y & =-A_{\zeta} X+\frac{1}{X}
\end{aligned}
$$

Received December 9, 1999.

2000 Mathematics Subject Classification. 53B20, 53B25, 53C55.

Key words and phrases. Kaehler manifold, CR-submanifold, Sasakian anti-holomorphic submanfold. 
for any $X, Y \in \Gamma(T M)$ and $\zeta \in \Gamma\left(T M^{\perp}\right)$, where $T M^{\perp}$ denote the normal bundle of $M$ in $N, \nabla^{\perp}$ the normal connection on $T M^{\perp}$ and $h$ the second fundamental form of $M$. The fundamental tensor of Weingarten $A_{\zeta}$ is related to $h$ by

$$
\left\langle A_{\zeta} X, Y\right\rangle=\langle h(X, Y), \zeta\rangle
$$

Let $R$ and $R^{\perp}$ be the curvature tensors associated with $\nabla$ and $\nabla^{\perp}$ respectively. If $R=0$ then $M$ is called a locally Euclidean space and if $R^{\perp}=0$ then we say that the normal connection $\nabla^{\perp}$ is flat. A normal vector field $\zeta$ is said to be parallel if we have $\nabla_{X}^{\perp} \zeta=0$, for any $X \in \Gamma(T M)$.

A distribution $F$ on $M$ is said to be auto-parellel if we have $\nabla_{X} Y \in \Gamma(F)$, for any $X, Y \in \Gamma(F)$. It is not hard to see that a distribution is auto-parallel if and only if it is integrable and each of its leaf is totally geodesic in $M$. Also, a distribution $F$ is parallel if and only if both $F$ and $F^{\perp}$ are auto-parallel, where $F^{\perp}$ is the complementary orthogonal distirbution to $F$. In this situation, we say that $M$ is locally a Riemannian product $M^{\prime} \times M^{\prime \prime}$, where $M^{\prime}$ and $M^{\prime \prime}$ are the leaves of $F$ and $F^{\perp}$ respectively.

Now suppose $N$ is a Kaehler manifold with complex structure $J$, i.e.,

$$
\left(\tilde{\nabla}_{X} J\right) Y=0, \quad \text { for } X, Y \in \Gamma(T N)
$$

If there exists on $M$ a holomorphic distribution $D$ such that its complementary orthogonal distribution $D^{\perp}$ is anti-invariant (i.e., $J D_{x}=D_{x}$ and $J D_{x}^{\perp} \subseteq T_{x}^{\perp} M, x \in M$ ), then $M$ is called a $C R$-submanifold of $N$ (see [1]). If $J D_{x}=T_{x}^{\perp} M$ is called an anti-holomorphic submanifold. For each $X \in \Gamma(T M)$, we put

$$
J X=\phi X+\omega X
$$

where $\phi X$ is the tangential part and $\omega X$ is normal part of $J X$. Similarly, we put

$$
J \zeta=B \zeta+C \zeta, \quad \text { for } \zeta \in \Gamma\left(T M^{\perp}\right)
$$

where $B \zeta$ is the tangential part and $C \zeta$ is the normal part of $J \zeta$.

Next, let us recall the definition of a Sasakian manifold. Let $M$ be a Sasakian manifold with Sasakian structure $(\phi, \xi, \eta,\langle\rangle$,$) . Then they satisfy (see [7])$

$$
\begin{aligned}
\phi^{2} X & =-X+\eta(X) \xi, \quad \eta(\xi)=1, \\
\langle\phi X, \phi Y\rangle & =\langle X, Y\rangle-\eta(X) \eta(Y), \\
\left(\nabla_{X} \phi\right) Y & =\langle X, Y\rangle \xi-\eta(Y) X,
\end{aligned}
$$

for any $X, Y \in \Gamma(T M)$. The following theorem is known (see [5]).

Theorem 2.1. Let $M$ be a Riemannian manifold. If $M$ admits a killing vector field $\xi$ of constant length satisfying

$$
\lambda^{2}\left(\nabla_{X} \nabla_{Y} \xi-\nabla_{\nabla_{X} Y} \xi\right)=\langle Y, \xi\rangle X-\langle X, Y\rangle \xi
$$


for a non-zero constant $\lambda$ and $X, Y \in \Gamma(T M)$, then $M$ is homothetic to a Sasakian manifold.

\section{Sasakian Anti-Homorphic Submanifold}

Let $M$ be a CR-submanifold of a Kaehler manifold $N$. For any $U, V \in \Gamma(T M)$, we put

$$
S(U, V)=[\phi, \phi](U, V)-2 B d \omega(U, V) .
$$

Here $[\phi, \phi]$ is the Nijenhuis tensor field of $\phi$ defined by

$$
[\phi, \phi](U, V)=[\phi U, \phi V]+\phi^{2}[U, V]-\phi[U, \phi V]-\phi[\phi U, V]
$$

and $d \omega$ is the exterior derivative of $\omega$ with

$$
d \omega(U, V)=\frac{1}{2}\left[\nabla_{U}^{\perp} \omega V-\nabla_{V}^{\perp} \omega U-\omega[U, V]\right] .
$$

The CR-submanifold $M$ is said to be normal if the tensor field $S$ vanishes identically on $M$.

The following theorem characterizes a normal CR-submanifold of a Kaehler manfold (see $[3])$.

Theorem 3.1. The CR-submanifold $M$ of a Kaehler manifold $N$ is normal if and only if

$$
A_{\omega Z} \phi X=\phi A_{\omega Z} X
$$

for any $X \in \Gamma(D)$ and $Z \in \Gamma\left(D^{\perp}\right)$.

Corollary 3.2. The CR-submanifold $M$ of a Kaehler manfold $N$ is normal if and only if

$$
B h(U, \phi V)+B h(\phi U, V)=0
$$

for any $U, V \in \Gamma(T M)$.

Proof. Let $P$ and $Q$ be the projection of $T M$ onto $D$ and $D^{\perp}$ respectively. Then for any $U, V \in \Gamma(T M)$ and $Z \in \Gamma\left(D^{\perp}\right)$ we have

$$
\begin{aligned}
\left\langle A_{\omega Z} \phi U, V\right\rangle-\left\langle\phi A_{\omega Z} U, V\right\rangle & =\left\langle A_{\omega Z} \phi P U, V+A_{\omega Z} \phi Q U, V\right\rangle-\left\langle\phi A_{\omega Z} P U-\phi A_{\omega Z} Q U, V\right\rangle \\
& =\left\langle A_{\omega Z} \phi P U, V-\phi A_{\omega Z} P U, V\right\rangle-\left\langle Q U, A_{\omega Z} \phi P V-\phi A_{\omega Z} P V\right\rangle .
\end{aligned}
$$

It follows from Theorem 3.1 that $M$ is normal if and only if

$$
A_{\omega Z} \phi U-\phi A_{\omega Z} U=0 .
$$

On the other hand, we have

$$
\langle B h(U, \phi V)+B h(\phi U, V), Z\rangle=-\left\langle A_{\omega Z} \phi U-\phi A_{\omega Z} U, V\right\rangle .
$$


Together with (3) we obtain (2).

If $M$ is an anti-holomorphic submanifold, then $J \zeta=B \zeta$, for any $\zeta \in \Gamma\left(T M^{\perp}\right)$. Thus, it follows from Corollary 3.2 that we have the following

Corollary 3.3. The anti-holomorphic submanifold $M$ of a Kaehler manfold $N$ is normal if and only if

$$
h(U, \phi V)+h(\phi U, V)=0
$$

for any $U, V \in \Gamma(T M)$.

Let $\left\{F_{1}, \ldots, F_{p}, J F_{1}, \ldots, J F_{p}\right\},(2 p=\operatorname{dim} D)$, be an arbitrary local field of orthonormal frames on $D$. We define the $D$-mean curvature vector $H_{D}$ of $M$ by

$$
H_{D}=\frac{1}{2 p} \sum_{k=1}^{P}\left(h\left(F_{k}, F_{k}\right)+h\left(J F_{k}, J F_{k}\right)\right) .
$$

We say that an anti-holomorphic submanifold $M$ is contact if $H_{D} \neq 0$ and for any $U, V \in \Gamma(T M)$ we have

$$
d \omega(U, V)=-\langle U, \phi V\rangle H_{D}
$$

or equivalently,

$$
h(\phi U, V)-h(U, \phi V)=-2\langle U, \phi V\rangle H_{D} .
$$

A Sasakian CR-submanifold is normal contact CR-submanifold of $N$.

The following characterization theorem plays a fundamental role in this paper.

Theroem 3.4. Let $M$ be an anti-holomorphic submanifold of $N$. If $H_{D} \neq 0$, then $M$ is a Sasakian anti-homorphic submanifold if and only if

$$
h(X, V)=\langle X, V\rangle H_{D}
$$

for any $V \in \Gamma(T M)$ and $X \in \Gamma(D)$.

Proof. If $M$ is a Sasakian anti-homolorphic submanifold, then by using Corollary 3.3 and (5) we have

$$
h(\phi U, V)=\langle\phi U, V\rangle H_{D}
$$

for any $U, V \in \Gamma(T M)$. In particular, if we put $X=\phi U$ then (6) is obtained.

Conversely, for any $U, V \in \Gamma(T M)$, since $\phi U \in \Gamma(D)$ we have

$$
h(\phi U, V)=\langle\phi U, V\rangle H_{D} .
$$

By a direct computation, we can see that conditions (4) and (5) are satisfied. Accordingly, $M$ is Sasakian.

The following result on a Sasakian anti-holomorphic submanifold with flat normal connection is due to Sun-Li [6]. 
Theorem 3.5. Let $M$ be an anti-holomorphic submanifold of $N$ with flat normal connection. If $\operatorname{dim} D \geq 4$, then the $D$-mean curvature tensor $H_{D}$ is parallel.

Form now on, we assume that $M$ is a connected Sasakian anti-submanifold of a Kaehler manifold $N$ with flat normal connection and with $\operatorname{dim} D \geq 4$. Then $H_{D}$ is parallel by Theorem 3.5. If we put $\mu=\left\|H_{D}\right\|$, then

$$
X \mu^{2}=X\left\langle H_{D}, H_{D}\right\rangle=2\left\langle\nabla_{x}^{\perp} H_{D}, H_{D}\right\rangle=0, \quad \text { for } X \in \Gamma(T M) .
$$

This means that, $\mu^{2}$ is a constant on some open subset of $M$ and so is $\mu$. As $M$ is connected and $H_{D} \neq 0, \mu$ is a non-zero constant defined on $M$ and hence $\xi=\frac{1}{\mu} J H_{D}$ is unit vector field in $D^{\perp}$ defined on the whole of $M$. Furthermore, for any $U \in \Gamma(T M)$, since $H_{D}$ is parallel we have

$$
\nabla_{U}^{\perp} J \xi=\nabla_{U}^{\perp} J\left[\frac{1}{\mu} J H_{D}\right]=-\frac{1}{\mu} \nabla_{U}^{\perp} H_{D}=0 .
$$

It follows that $J \xi$ is also a parallel normal vector field.

Next, we define a distribution $F$ on $M$ by

$$
F: x \rightarrow D_{x} \oplus\left\{\xi_{x}\right\}, \quad \text { for } x \in M
$$

where $\left\{\xi_{x}\right\}$ is the vector subspace of $T_{x} M$ spanned by $\xi_{x}$. Denote by $F^{\perp}$ the complementary orthogonal distribution to $F$.

For each $Z \in \Gamma(F)$ we put

$$
\eta(Z)=\langle Z, \xi\rangle
$$

Then we have

$$
Z=P Z+\eta(Z) \xi
$$

We now prove a useful lemma.

Lemma 3.6. $\nabla_{U} \xi=-\mu \phi U$, for any $U \in \Gamma(T M)$.

Proof. For any $U, V \in \Gamma(T M)$, since $N$ is Kaehlerian we have

$$
\left\langle\left(\tilde{\nabla}_{U} J\right) J \xi, V\right\rangle=0 .
$$

By using the fact $J \xi$ is parallel, and together with the Gauss and Weingarten formulas, this yields

$$
-\left\langle\nabla_{U} \xi, V\right\rangle+\left\langle\phi A_{J \xi} U, V\right\rangle=0
$$

Now, by using (1) and Theorem 3.4 we get

$$
\left\langle\nabla_{U} \xi, V\right\rangle=\langle-\mu \phi U, V\rangle
$$

Hence, the lemma is proved. 
We now consider the following local field of orthonormal frames on $D^{\perp}$

$$
\left\{\xi=E_{1}, E_{2}, \ldots, E_{q}\right\}, \quad\left(q=\operatorname{dim} D^{\perp}\right)
$$

such that each $J E_{i}$ is a parallel normal vector field. The existence of such a distinguished field of frames is assured by [4, Prop. 1.1 and Prop. 1.3 of Chap.4]. Then we have the following

Lemma 3.7. $\nabla_{E_{j}} E_{i}=0$, for $i, j=1,2, \ldots, q$.

Proof. First, for any $X \in \Gamma(D)$ we have

$$
\left\langle-A_{J E_{i}} E_{j}-J \nabla_{E_{j}} E_{i}, X\right\rangle=\left\langle\left(\tilde{\nabla}_{E_{j}} J\right) E_{i}, X\right\rangle=0 .
$$

It follows from (1) and Theorem 3.4 that we obtain

$$
\left\langle J \nabla_{E_{j}} E_{i}, X\right\rangle=\left\langle X, E_{j}\right\rangle\left\langle H_{D}, J E_{i}\right\rangle=0 .
$$

Therefore, $\nabla_{E_{j}} E_{i} \in \Gamma\left(D^{\perp}\right)$. Moreover, taking into account that $J E_{i}$ is parallel and $\left\langle\left(\tilde{\nabla}_{E_{j}} J\right) E_{i}, J E_{k}\right\rangle=0$, we obtain $\left\langle\nabla_{E_{j}} E_{i}, E_{k}\right\rangle=0$. Accordingly, $\nabla_{E_{j}} E_{i}=0$.

Proposition 3.8. The distributions $F$ and $F^{\perp}$ are auto-parallel and consequently are integrable.

Proof. For any $Z, W \in \Gamma(F)$ and $j,(2 \leq j \leq q)$, we have

$$
\begin{aligned}
\left\langle\nabla_{Z} W, E_{j}\right\rangle & =\left\langle\nabla_{Z} P W, E_{j}\right\rangle+\left\langle\nabla_{Z}(\eta(W) \xi), E_{j}\right\rangle \\
& =\left\langle\nabla_{Z} P W, E_{j}\right\rangle+\eta(W)\left\langle-\mu \phi Z, E_{j}\right\rangle, \quad \text { by Lemma } 3.6 \\
& =\left\langle\nabla_{Z} P W, E_{j}\right\rangle
\end{aligned}
$$

On the other hand, we have

$$
\left\langle h(Z, \phi P W)-J \nabla_{Z} P W, J E_{j}\right\rangle=\left\langle\left(\tilde{\nabla}_{Z} J\right) P W, J E_{j}\right\rangle=0 .
$$

It follows that

$$
\left\langle\nabla_{Z} P W, E_{j}\right\rangle=\left\langle h(Z, \phi W), J E_{j}\right\rangle=\langle Z, \phi W\rangle\left\langle H_{D}, J E_{j}\right\rangle=0
$$

since $H_{D}=-\mu J \xi \perp J E_{j}$. Hence, $\nabla_{Z} W \in \Gamma(F)$, i.e., $F$ is auto-parallel.

In order to prove $F^{\perp}$ is auto-parallel, it suffices to shows that $\nabla_{E_{j}}\left(f E_{i}\right) \in \Gamma\left(F^{\perp}\right)$, for $i, j \geq 2$ and for any differentiable function $f$ on $M$. Since $\nabla_{E_{j}} E_{i}=0$, we obtain $\nabla_{E_{j}}\left(f E_{i}\right)=\left(E_{j} f\right) E_{i} \in \Gamma\left(F^{\perp}\right)$.

We are now ready to prove the main result of this paper.

Theorem 3.9. Let $M$ be a connected Sasakian anti-holomorphic submanifold of a Kaehler manifold $N$ with flat normal connection and with $\operatorname{dim} D \geq 4$. Then $M$ is locally 
a Riemannian product $M^{\prime} \times M^{\prime \prime}$, where $M^{\prime}$ is homothetic to a Sasakian manifold and $M^{\prime \prime}$ is a locally Euclidean space.

Proof. Let $M^{\prime}$ and $M^{\prime \prime}$ be the leaves of $F$ and $F^{\perp}$ respectively. Since both $F$ and $F^{\perp}$ are auto-parallel, $M$ is locally a Riemannian product $M^{\prime} \times M^{\prime \prime}$. Moreover, since $M^{\prime \prime}$ is totally geodesic in $M$, form the Gauss formulas and Lemma 3.7 we have

$$
\nabla_{E_{j}}^{\prime \prime} E_{i}=\nabla_{E_{j}} E_{i}=0
$$

where $\nabla^{\prime \prime}$ is the Levi-Civita connection induced by $\nabla$ on $M^{\prime \prime}$. It follows that

$$
R^{\prime \prime}\left(E_{i}, E_{j}\right) E_{k}=0
$$

where $R^{\prime \prime}$ is the Riemannian curvature on $M^{\prime \prime}$ and $i, j, k \geq 2$. Consequently, $M^{\prime \prime}$ is a locally Euclidean space.

Next, denote by $\nabla^{\prime}$ the Levi-Civita connection induced by $\nabla$ on $M^{\prime}$. Taking into account that $M^{\prime}$ is totally geodesic in $M$, from the Gauss formulas, for any $Z, W \in \Gamma(F)$ we have

$$
\nabla_{Z}^{\prime} W=\nabla_{Z} W=P \nabla_{Z} W+\eta\left(\nabla_{Z} W\right) \xi
$$

It follows from Lemma 3.6 and (7) that we obtain

$$
\nabla_{Z}^{\prime} \nabla_{W}^{\prime} \xi-\nabla_{\nabla_{Z}^{\prime} W}^{\prime} \xi=-\mu\left(P \nabla_{Z} \phi W-\phi \nabla_{Z} W\right)-\mu^{2}\langle\phi W, \phi Z\rangle \xi
$$

On the other hand we can see

$$
\left\langle P \nabla_{Z} \phi W-P A_{\omega W} Z-\phi \nabla_{Z} W, X\right\rangle=\left\langle\left(\tilde{\nabla}_{Z} J\right) W, X\right\rangle=0, \quad \text { for } X \in \Gamma(D) .
$$

Together with Theorem 3.4, we obtain

$$
P \nabla_{Z} \phi W-\phi \nabla_{Z} W=-\mu\langle\xi, W\rangle P Z .
$$

Therefore, (8) becomes

$$
\begin{aligned}
\nabla_{Z}^{\prime} \nabla_{W}^{\prime} \xi-\nabla_{\nabla_{Z}^{\prime} W}^{\prime} \xi & =\mu^{2}\langle\xi, W\rangle P Z-\mu^{2}\langle\phi W, \phi Z\rangle \xi \\
& =\mu^{2} \eta(W) P Z-\mu^{2}\langle W, Z\rangle \xi+\mu^{2} \eta(W) \eta(Z) \xi \\
& =\mu^{2}\{\eta(W) Z-\langle W, Z\rangle \xi\} .
\end{aligned}
$$

Furthermore, by using Lemma 3.6 and (7) we can see

$$
\left\langle\nabla_{Z}^{\prime} \xi, W\right\rangle+\left\langle Z, \nabla_{W}^{\prime} \xi\right\rangle=0, \quad \text { for any } Z, W \in \Gamma(F)
$$

which means $\xi$ is a killing vector field on $M^{\prime}$. Hence, $M^{\prime}$ is homothetic to a Sasakian manifold by means of Theorem 1.1 and this completes the proof. 


\section{References}

[1] Bejancu, CR-submanifolds of a Kaehler manifold I, Proc. Amer Math. Soc., 69(1978), 134-142.

[2] Bejancu A., Geometry of CR-submanifolds, Reidel Holland, 1986.

[3] Bejancu A., Normal CR-submanifolds of Kaehler manifolds, Ann. Univ. Al. I. Cuza, Iasi., 26(1980), 123-132.

[4] Chen, B. Y., Geometry of submanifolds, M. Dekker Inc. New York, 1973.

[5] Okumura, M., Cetain almost contact hypersurfaces in Kaehlerian manifolds of constant holomorphic sectional curvatures, Tohoku Math. J., 16(1964), 270-284.

[6] Sun, Z. Z. and Li, H. Z., Sasakian submanifolds of Kaehler monifolds, Adv. In Math. (China), 20(1991), 363-370.

[7] Yano, K. and Kon, M., structure on maniofolds, Series in Pure Math., 3, World Sci. Publ. Co., Singapore, 1984.

School of Arts and Science, Tunku Abdul Rahman College, P. O. Box 10979, 50932, Kuala Lumpur, Malaysia. 